\title{
Deformation invariant optical processors using coordinate transformations
}

\author{
Demetri Psaltis and David Casasent
}

\begin{abstract}
A general formulation of the properties, optical synthesis, and existence of space variant optical processors using coordinate transformations is provided. General expressions that the coordinate transformations must satisfy are described. Specific examples of the use of the methodology to determine the existence of and methods for the realization of space variant optical processors are included for various specific data deformations and applications.
\end{abstract}

\section{Introduction}

The advantages of optical processing, a description of the various operations possible, and many applications of optical processors have been described elsewhere. ${ }^{1-3}$ We are presently concerned with the use of space variant optical processors to increase the flexibility, practicality, and utility of optical processors. Previous approaches to extend the repertoire of operations possible in an optical processor have included hybrid optical/digital processors, ${ }^{4-6}$ optical feedback systems, ${ }^{7}$ processing using halftone screens, ${ }^{8}$ use of computer generated holograms, ${ }^{9,10}$ image restoration by coordinate transformations, ${ }^{11}$ generalized optical operators, ${ }^{12,13}$ nonlinear operations, ${ }^{14,15}$ use of Mellin transforms, ${ }^{16-19}$ and other methods.

Most of these methods involve the synthesis of various types of space variant optical processors. Several general formulations have been provided for frequency variant systems ${ }^{20}$ for space variant systems using volume holographic ${ }^{21}$ and holographic ${ }^{22}$ representations and for specific applications such as image restoration ${ }^{11}$ and shear interferometry. ${ }^{10}$ In this paper, we consider a general formulation of deformation-invariant, space-variant optical processors using coordinate transformations. This treatment will emphasize the correlation and pattern recognition applications and the optical synthesis of such space variant systems for arbitrarily deformed general functions. Other papers on space variance by coordinate transformations ${ }^{11}$ address the analysis of imaging systems by decomposition of the system's action into a coordinate transformation and a space invariant system.

\footnotetext{
The authors are with Carnegie-Mellon University, Department of Electrical Engineering, Pittsburgh, Pennsylvania 15213.

Received 16 February 1977.
}

General expressions will be derived that the deformation and coordinate transformation must satisfy for the case of a general distortion if one is to convert a space invariant optical system into a space variant one by coordinate transformation. Specific examples are included to demonstrate the use of this formulation for several practical applications. This general formulation of the solution to deformation invariant correlation should enable other researchers to apply these techniques we have developed to their specific data distortion problems. Although the treatment to follow emphasizes the optical realization of such systems, their implementation by special purpose solid state, CCD, $\mathrm{CID}$, acoustooptic, and digital processors is likewise possible from the formulations to be presented.

\section{Space Variant Correlation}

Since pattern recognition by correlation represents one of the most powerful applications of coherent optical processing, we choose to emphasize such systems in this general analysis. In most practical optical pattern recognition correlators, the input and reference functions will be deformed versions of one another. We will show by specific examples that many practical deformations can be described simply by transformations of the coordinates of the input functions. In these practical situations, conventional optical correlations will not yield acceptable results, and a space-variant correlator invariant to the input deformation is needed. One successful formulation of such a system that we consider is the use of coordinate transformations on the input data. These coordinate transformed data then serve as the inputs to a conventional space invariant correlator, and an over-all space variant system (invariant to the input deformation) results when the input coordinate transformation is properly chosen. 
A block diagram of a general space variant correlator is shown in Fig. 1. The undeformed or reference function $f(x)$ degraded by the generalized deformation function $g(x, a)$ is

$$
f[g(x, a)]=f\left(x^{\prime}\right)=f^{\prime}(x) .
$$

$x^{\prime}=g(x, a)$ describes the deformation as a function of both the input coordinate and a distortion parameter $a$. To correlate the reference $f(x)$ and input $f^{\prime}(x)$ functions in a conventional space invariant system, a coordinate transformation $h^{-1}(x)=\zeta$ is applied to both functions producing the two coordinate transformed functions $f[h(\zeta)]=f_{1}(\zeta)$ and $f^{\prime}[h(\zeta)]=f_{1}{ }^{\prime}(\zeta)$. These new functions must be shifted versions of one another, i.e.,

$$
f_{1}(\zeta)=f_{1}{ }^{\prime}\left(\zeta-\zeta_{0}\right)
$$

if they are to be correlated using a space invariant system. The shift parameter $\zeta_{0}$ must be a constant dependent only on the deformation parameter $a$.

If $\zeta=h^{-1}(x)$ is a one-to-one correspondence function (and thus has an inverse), the functions $f_{1}(\zeta)$ and $f_{1}{ }^{\prime}(\zeta)$ will correlate if and only if $f(x)$ and $f^{\prime}(x)$ are related by Eq. (1). The system thus tests the validity of Eq. (1) and recognizes the deformed function $f^{\prime}(x)$ independent of the value of the deformation parameter $a$.

\section{Determination of the Coordinate Transformation}

The general expression that the deformation $g(x, a)$ and coordinate transformation $x=h(\zeta)$ must satisfy follows from Eqs. (1) and (2) to be

$$
g[h(\zeta), a]=h\left(\zeta-\zeta_{0}\right)
$$

If a function $h(\zeta)$ satisfying Eq. (3) can be found, the effect of the deformation can be converted to a shift in $\zeta$, and a space variant system can be used to correlate $f(\zeta)$ and $f^{\prime}(\zeta)$. We represent this more clearly by taking the inverse transform $h^{-1}$ of Eq. (3) and using Eq. (1) to obtain

$$
h^{-1}(x)-h^{-1}\left(x^{\prime}\right)=\zeta_{0}
$$

which clearly indicates that the transformation $h^{-1}$ converts the deformation into a shift by $\zeta_{0}$ in the transformed coordinates $\zeta$.

A general expression for $h^{-1}$ can be derived by forming the derivative of Eq. (4) with respect to $a$.

$$
\frac{d h^{-1}\left(x^{\prime}\right)}{d x^{\prime}} \cdot \frac{d x^{\prime}}{d a}=\frac{d \zeta_{0}}{d a}
$$

from which we obtain the desired general equation

$$
h^{-1}(x)=-\frac{d \zeta_{0}}{d a} \int_{-\infty}^{x} \frac{d x^{\prime}}{\left(\frac{d x^{\prime}}{d a}\right)}=\frac{d \zeta_{0}}{d a} \int_{-\infty}^{x} \frac{\frac{\partial g(x, a)}{\partial x}}{\frac{\partial g(x, a)}{\partial a}} d x .
$$

If a function $\zeta_{0}$ of $a$ only can be found such that $h^{-1}(x)$ defined by Eq. (6) is independent of $a$, this coordinate transformation can be used to produce a space variant system as shown in Fig. 1 that is invariant to the deformation $g(x, a)$.

The intensity of the correlation peak will be the same as that of the autocorrelation of the reference function.

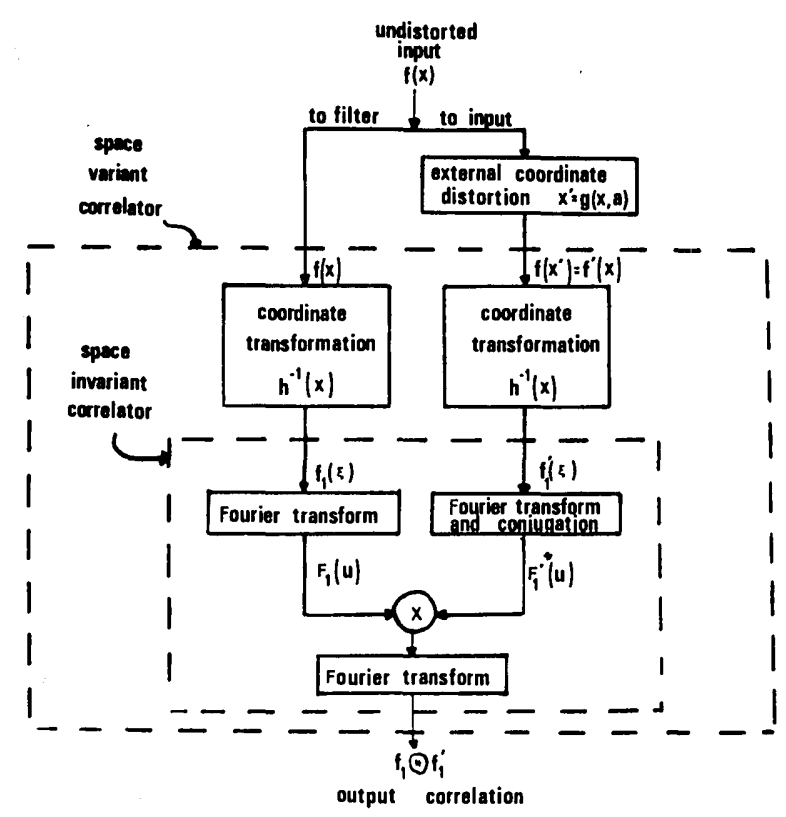

Fig. 1. Functional block diagram of a general space-variant, deformation-invariant correlator.

Furthermore, by converting the deformation to a shift in the $\zeta$ coordinates, the location of the output correlation peak (which is proportional to the shift $\zeta_{0}$ in the input to a space invariant correlator) will be related to the deformation parameter $a$. (Recall $\zeta_{0}$ is a function of $a$ only.) The deformation parameter $a$ can thus be found from the location of the output correlation peak with no $a$ priori knowledge of $a$ assumed.

In the preceding general analysis, deformations affecting one input coordinate were considered for simplicity. For the more general case of an $n$th order distortion function $g_{n}\left(x, a_{n}\right)$, each coordinate can be treated independently if all $g_{n}$ deforming functions affect only one coordinate. If any one deforming function depends on more than one coordinate, the analysis method must be modified.

For the case of two deforming functions $g_{x}\left(x, y, a_{x}, a_{y}\right)$ and $g_{y}\left(x, y, a_{x}, a_{y}\right)$, a pair of coordinate transformations $\zeta=h_{x}(x, y)$ and $\eta=h_{y}(x, y)$, where the coordinates of the function in the coordinate transformed plane are $(\zeta, \eta)$, are required. These transformations must satisfy the differential equations

$$
\begin{gathered}
\frac{\partial h_{x}\left(x^{\prime}, y^{\prime}\right)}{\partial x^{\prime}} \frac{\partial x^{\prime}}{\partial a_{x}}+\frac{\partial h_{x}\left(x^{\prime}, y^{\prime}\right)}{\partial y^{\prime}} \frac{\partial y^{\prime}}{\partial a_{x}}=\frac{\partial \zeta_{0}\left(a_{x}\right)}{\partial a_{x}}, \\
\frac{\partial h_{x}\left(x^{\prime}, y^{\prime}\right)}{\partial x^{\prime}} \frac{\partial x^{\prime}}{\partial a_{y}}+\frac{\partial h_{x}\left(x^{\prime}, y^{\prime}\right)}{\partial y^{\prime}} \frac{\partial y^{\prime}}{\partial a_{y}}=\frac{\partial \zeta_{0}\left(a_{x}\right)}{\partial a_{y}}=0, \\
\frac{\partial h_{y}\left(x^{\prime}, y^{\prime}\right)}{\partial x^{\prime}} \frac{\partial x^{\prime}}{\partial a_{x}}+\frac{\partial h_{y}\left(x^{\prime}, y^{\prime}\right)}{\partial y^{\prime}} \frac{\partial y^{\prime}}{\partial a_{x}}=\frac{\partial \eta_{0}\left(a_{y}\right)}{\partial a_{x}}=0, \\
\frac{\partial h_{y}\left(x^{\prime}, y^{\prime}\right)}{\partial x^{\prime}} \frac{\partial x^{\prime}}{\partial a_{y}}+\frac{\partial h_{y}\left(x^{\prime}, y^{\prime}\right)}{\partial y^{\prime}} \frac{\partial y^{\prime}}{\partial a_{y}}=\frac{\partial \eta_{0}\left(a_{y}\right)}{\partial a_{y}}
\end{gathered}
$$




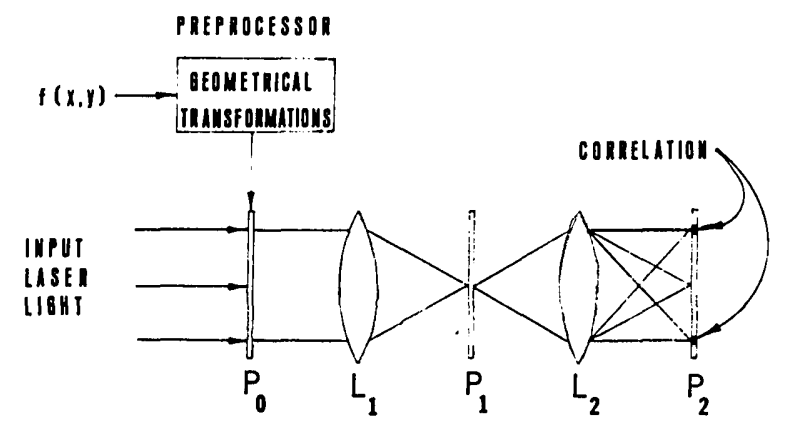

Fig. 2. Schematic diagram of a general optical space-variant, deformation-invariant optical correlator.

where the input coordinates of the deformed function are $\left(x^{\prime}, y^{\prime}\right)$, and the deformations are converted to shifts by $\zeta_{0}$ and $\eta_{0}$, where $\zeta_{0}$ and $\eta_{0}$ are dependent only on the deformation parameters $a_{x}$ and $a_{y}$, respectively [see Eqs. (7b) and (7c)]. The coordinates of the peak of the correlation of these coordinate transformed functions $f_{1}(\zeta, \eta)$ and $f_{1}{ }^{\prime}(\zeta, \partial)$ are now $\zeta_{0}$ and $\eta_{0}$. By analogy with the 1-D case and by virtue of the differential equations described, the deformation parameters $a_{x}$ and $a_{y}$ can be determined from the location of the output correlation peak.

\section{Implementation}

We now consider the practical implementation details of a space variant and deformation invariant correlator of the type discussed above. The motivation for the formulation given for the space variant correlator architecture chosen should be apparent. By fabricating the space variant correlator as a coordinate transformation followed by a conventional space invariant correlator, the system can be realized using conventional optical correlators. This allows one to utilize the parallel processing, 2-D nature, high speed, and Fourier transform properties of a coherent optical processor while expanding its flexibility and practicality. This architectural design philosophy also allows realization of a space-variant system using digital, CCD, solid-state, and other correlation techniques.

The schematic diagram of a general optical space variant correlator is shown in Fig. 2. A matched spatial filter or frequency plane correlator ${ }^{21}$ schematic is shown although a joint transform optical correlator ${ }^{22}$ and other optical correlator configurations can be employed. As before, we denote the reference or undeformed function by $f(x, y)$. The optical matched spatial filter of $f_{1}\left(\zeta_{1}, \zeta_{2}\right)$ is formed at plane $P_{1}$ by placing the coordinate transformed function $f_{1}\left(\zeta_{1}, \zeta_{2}\right)$ at the input plane $P_{0}$ and recording the interference of its Fourier transform (formed by lens $L_{1}$ at plane $P_{1}$ ) and a plane wave reference beam (shown at an angle $\theta$ to the optical axis in Fig. 2). The term of interest in the subsequent transmittance of plane $P_{1}$ is $F_{1}{ }^{*}(u, v)$ or the complex conjugate of the Fourier transform of $f_{1}\left(\zeta_{1}, \zeta_{2}\right)$ which diffracts light at an angle $\theta$ to the optical axis as shown by lens $L_{2}$ and plane $P_{2}$.

To correlate the two functions $f_{1}{ }^{\prime}\left(\zeta_{1}, \zeta_{2}\right)$ is placed at plane $P_{0}$ and the matched filter $F_{1}{ }^{*}(u, v)$ at plane $P_{1}$.
The light distribution incident on plane $P_{1}$ is then $F_{1}{ }^{\prime}(u, v)$, and the subsequent pattern transmitted through $P_{1}$ (at an angle $\theta$ to the optical axis, as shown in Fig. 2) is $F_{1}{ }^{\prime} F_{1}{ }^{*}$. Lens $L_{2}$ forms the Fourier transform of the product of two transforms at plane $P_{2}$ where the recorded light distribution is thus the correlation $f_{1}{ }^{\prime}\left(\zeta_{1}, \zeta_{2}\right) * f_{1}\left(\zeta_{1}, \zeta_{2}\right)$ of the two functions, as desired.

Two methods have been suggested for realizing the $h^{-1}(x)=\zeta$ input coordinate transformation preprocessing step. Both are compatible with the use of real-time optical spatial light modulators at planes $P_{0}$ and $P_{1}$. If the input light modulator is sequentially addressed by a scanning electron beam or laser beam, ${ }^{23}$ the required coordinate transformation can be realized by use of electronic analog modules to modify the sweep of the input light modulator or input pickup device. Examples of such systems for specific input deformations have been described previously. ${ }^{16,18}$ If the input spatial light modulator is optically addressed in parallel, ${ }^{23}$ the input coordinate transformation can be realized in parallel on the input data using computer generated hologram masks. This method has also been demonstrated..$^{19.24}$

\section{Examples}

Several specific examples will now be presented to demonstrate the use of the previously developed general theoretical formulation of space variant and deformation invariant correlation by coordinate transformation.

\section{A. Shift Deformation}

Perhaps the simplest case to consider is an input deformation described by $x^{\prime}=g(x, a)=x-a$. This deformation is simply a shift in the location of the input function by $a$. Substitution into Eq. (6) yields the required coordinate transformation, which is simply $x=$ $h(\zeta)=\zeta$ with $\zeta_{0}=a$. This trivial case simply illustrates that a space invariant system is invariant to a shift in the coordinates of the input function and that the location of the output correlation peak is proportional to the shift in the input function. Thus, in this case no coordinate transformation is required.

\section{B. Scale Deformation}

A far more practical deformation is a scale change between the input and reference functions. In this case, $x^{\prime}=g(x, a)=a x$. After substitution into Eq. (6), we find the required coordinate transformation to be $h^{-1}(x)=\ln x=\zeta$ and $\zeta_{0}=\ln a$. In this instance, the combination of this logarithmic coordinate transformation and the Fourier transform of this coordinate transformed function produces a specific mathematical operation known as the Mellin transform. ${ }^{25}$ The results of an optical Mellin transform correlator are shown in Fig. 3. The input function chosen was a simple square [Fig. 3(a)] and a distorted version of it [Fig. 3(b)] with a $200 \%$ scale increase $(a=2)$. The cross sections of the optical autocorrelation pattern of the original square and the optical cross correlation of the two squares are shown ${ }^{17}$ in Figs. 3(c) and 3(d). The space variant correlator of Fig. 2 was used for these experiments and the 


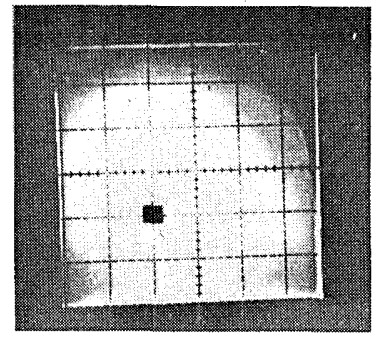

(a)

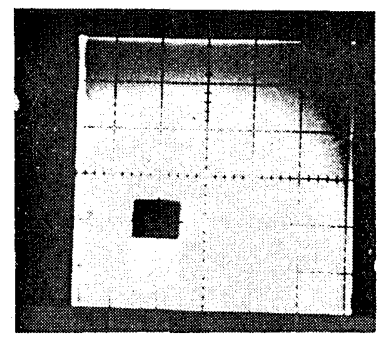

(b)

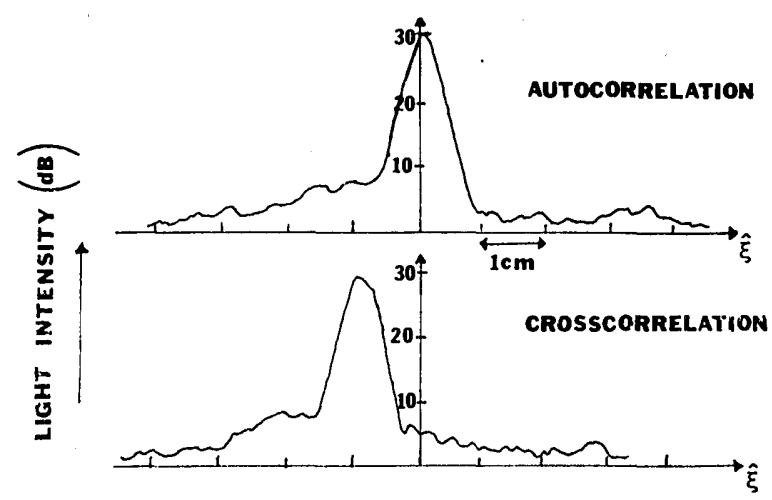

Fig. 3. Example of a scale-invariant, space-variant optical correlator: (a) undeformed input reference function $f(x)$; (b) deformed input function $f\left(x^{\prime}\right)=f(a x)$ with a $200 \%$ scale change distortion; (c) cross section of the autocorrelation; and (d) cross section of the cross correlation.

coordinate transformation $h^{-1}(x)=\ln x=\zeta$ realized by $\log$ modulating the deflection system of a CRT. From Figs. 3(c) and 3(d), we see that there is no SNR loss in the correlation and that the location of the cross-correlation peak has shifted from the location (in $\zeta$ space) of the autocorrelation peak by an amount $\zeta_{0}=\ln a$ proportional to the input scale change as predicted by Eq. (4).

\section{Exponentiation Deformation}

Another deformation that has been considered is an exponentiation of the input coordinates, i.e., $x^{\prime}=g(x, a)$ $=x^{a}$. From Eq. (6), the required input coordinate transformation is $\zeta=\ln (\ln x)$ and can be realized by a sequence of logarithmic scalings. As a simple demonstration of the space variant correlation of such distorted functions, the undistorted and distorted grating inputs of Figs. 4(a) and 4(b) were prepared. The required logarithmic coordinate scaling was applied to both functions, a matched filter formed of one function and the space invariant correlator of Fig. 2 again used. The resultant autocorrelation and cross-correlation patterns were scanned, and results similar to those of Figs. 3(c) and 3(d) were obtained.

\section{Rotational Deformation}

One of the most common system alignment induced deformations is a rotation between the input and reference functions. This type of deformations simultaneously affects both coordinates of the input function.
The required input coordinate transformations for this case are found by solving the differential equations in Eq. (7) to be

$$
\begin{aligned}
& \zeta=r=h_{x}(x, y)=\left(x^{2}+y^{2}\right)^{1 / 2}, \\
& \eta=\theta=h_{y}(x, y)=\tan ^{-1}(y / x) .
\end{aligned}
$$

This transformation is equivalent to a cartesian-to-polar coordinate conversion of the input function, and thus we replace $(\zeta, \eta)$ by the more conventional $(r, \theta)$ polar coordinate variables. A detailed discussion of this particular space variant system was published earlier, ${ }^{18}$ but not in the general formulation terms used above.

\section{Discussion}

A general procedure for synthesis of a deformation invariant correlator using an input coordinate transformation and a conventional space invariant correlator has been presented. Specific examples were provided to clarify the use of this technique for other researchers. The extension to deformations dependent on both input coordinates was also included.

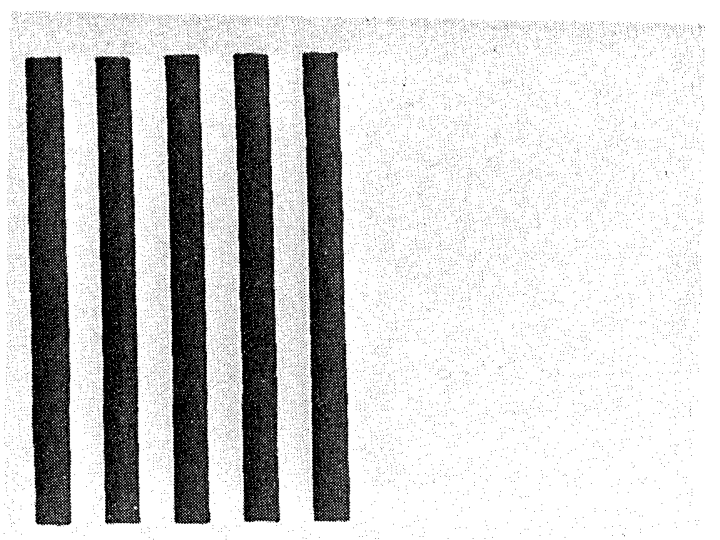

(a)

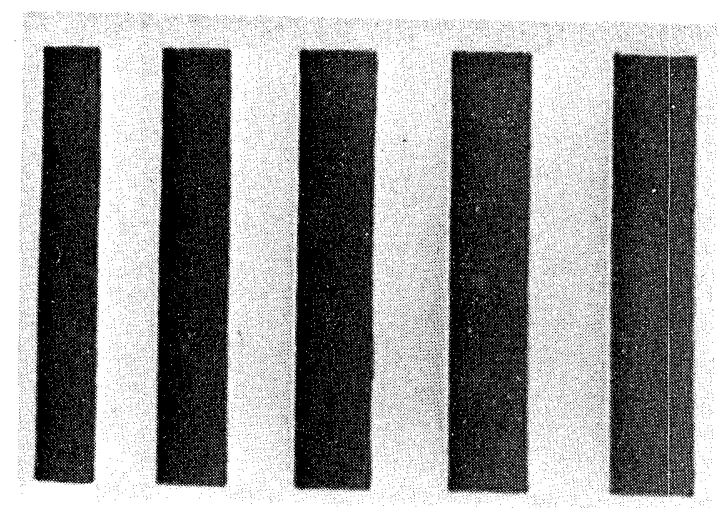

(b)

Fig. 4. Example of a deformation invariant correlator in which the input coordinate is exponentiated $x^{\prime}=g(x, a)=x^{a}$. (a) Reference input; (b) distorted input. The cross-sectional scans of the autocorrelations and cross-correlations are similar to those shown in Figs. $3(\mathrm{c})$ and $3(\mathrm{~d})$. 
Implicit in Eqs. (3)-(7) was the assumption that only one deformation parameter can be compensated for per geometrical axis by the methods described. When the input deformation depends on more than one parameter per axis, the input must be preprocessed to remove the effect of all deformation parameters in excess of one per axis. One method for achieving this is to first convert the effect of the deformation to a shift in the input using the methods described in Sec. II. The linear phase factor associated with the input shift can then be removed in the frequency plane. These multiple invariant cases and methods whereby this linear phase factor can be removed and the phase of the input function preserved will be published shortly.

The research support of the Office of Naval Research on contract NR 350-011, the AFOSR Air Systems Command on grant AFOSR-75-2851, and the Ballistic Missile Defense Advanced Technology Agency on contract DASG 60-77-C-0034 for various portions and applications of this work is gratefully acknowledged.

\section{References}

1. A. Van der Lugt, Proc. IEEE 62, 1300 (1974).

2. J. W. Goodman, Proc. IEEE 65, 29 (1977).

3. D. Casasent and H. J. Caulfield, Eds., Applications of Optical Data Processing (Springer-Verlag, Heidelberg, 1977).

4. D. Casasent, "Hybrid Processors," in Optical Data Processing, S. H. Lee, Ed. (Springer-Verlag, Heidelberg, 1977).

5. D. Casasent and W. Sterling, IEEE Trans. Comput. C-24, 348 (1975).

6. B. J. Thompson, Proc. IEEE 65, 62 (1977).

7. S. Lee, Opt. Eng. 13, 196 (1974).

8. S. Dashiel and A. Sawchuck, in Proceedings Electronic Optical Systems Design Conference Anaheim (1975) p. 9.

9. O. Bryngdahl, J. Opt. Soc. Am. 64, 1092 (1974).

10. O. Bryngdahl and W. H. Lee, J. Opt. Soc. Am. 64, 1606 (1974).

11. A. Sawchuck, J. Opt. Soc. Am. 64, 138 (1974).

12. F. P. Carlson, IEEE Intercom. Tech. Paper, 7/2 (March 1973).

13. P. Eguchi and F. P. Carlson, Appl. Opt. 9, 687 (1970).

14. K. Stalker and S. Lee, J. Opt. Soc. Am. 64, 564 (1974).

15. N. Farhat, IEEE Trans. Comput. C-24, 443 (1975).

16. D. Casasent and D. Psaltis, Opt. Eng. 15, 258 (1976).

17. D. Casasent and D. Psaltis, Opt. Commun. 17, 59 (1976).

18. D. Casasent and D. Psaltis, Appl. Opt. 15, 1795 (1976).

19. D. Casasent and D. Psaltis, Proc. IEEE 65, 77 (1977).

20. D. Casasent and A. Furman, Appl. Opt. 16, 1652 (1977).

21. A. Van der Lugt, IEEE Trans. Info. Theory, IT-10, 139 (1964).

22. J. Rau, J. Opt. Soc. Am. 57, 798 (1976).

23. D. Casasent, Proc. IEEE 65, 143 (1977).

24. D. Casasent and C. Szczutkowski, Opt. Commun. 19, 217 (1976).

25. R. Bracewell, The Fourier Transform and Its Applications (McGraw-Hill, New York, 1965), Chap. 12.

OPTICAL SOCIETY TO PRESENT MEGGERS AWARD

The Optical Society of America will present its 1977 William F. Meggers award jointly to Mark S. Fred and Frank S. Tomkins at the Society's annual meeting in Toronto, October 10-14.

Fred and Tomkins are scientists at the Argonne National Laboratory. Both their individual research accomplishments and their joint efforts in the development of the spectroscopic laboratory at Argonne into one of the world's finest facilities will be recognized by the award.

Fred's spectroscopic investigations span the whole periodic table, from hydrogen to fermium. His analysis of the actinide elements is well known. He received his graduate and undergraduate education in physics at the University of Chicago. In 1948 Fred joined the staff of the Argonne National Laboratory, where he is a senior research chemist. He is a Fellow of the Optical Society of America.

The development of the electrodeless lamp and the induction-heated absorption furnace are but two of Tomkins's contributions to the advancement of atomic spectroscopy. He received his undergraduate training in chemistry at Kalamazoo College and completed his graduate work at Michigan State University. He is a senior scientist at Argonne, where he has been employed since 1946. He is a Fellow of the Optical Society of America.

The William F. Meggers Award was established by the Optical Society in 1970 to honor the memory of Meggers and his notable contributions to the fields of spectroscopy and metrology. The award, which consists of a silver medal and a citation, is presented annually for outstanding work in spectroscopy. 